\title{
Desarrollo de competencias emprendedoras en docentes de universidades públicas autónomas
}

\author{
Hender Viloria, PhD $^{17}$ \\ Universidad del Zulia. \\ henderviloria@gmail.com
}

\author{
How to cite this paper: \\ Viloria Hender (2017) Desarrollo de \\ competencias emprendedoras en \\ docentes de universidades públicas \\ autónomas. Revista Encuentros, \\ Universidad Autónoma del Caribe, \\ vol. 15-01 pp.133-146 \\ DOI: http: / / dx.doi.org/10.15665/ re.v15i1.815
}

\section{RESUMEN}

En el presente artículo de investigación se analiza el desarrollo de competencias emprendedoras en docentes universidades públicas autónomas del estado Zulia, basado en las teorías de Auletta y Puente (2010), Mas Torelló (2011) e Hisrich, (2005) La investigación fue de tipo descriptiva, no experimental y de campo. Se aplicó un cuestionario tipo Likert a una muestra de 44 docentes-investigadores de universidades públicas autónomas del estado Zulia. Se obtuvo como resultados previos que hay un moderado nivel de actitud y atributos para el emprendimiento y un alto rango de competencias tecnológicas para transformar la realidad con conocimientos innovadores, pero con bajo nivel de financiamiento así como recursos escasos. Por lo que se propone cultivar a través de un modelo, el desarrollo competencias emprendedoras para la innovación en los docentes de centros de investigación, con el fin de transformar las universidades públicas autónomas en instituciones que produzcan resultados tangibles para el desarrollo socio-productivo de la nación.

Palabras clave: competencias emprendedoras, Innovación, Universidades Públicas Autónomas, Desarrollo socioeconómico. 


\title{
Development of Entrepreneurial skills for innovation in of autonomous public universities
}

\begin{abstract}
For this thesis we aim to propose a model for innovation entrepreneurship in humanistic core of autonomous public universities, bassed on the theory of Auletta y Puente (2010), Mas Torelló (2011) and Hisrich, (2005). It establishes as a fundamental characteristic for the development of entrepreneurship and social enterprise to build this core humanistic model of autonomous public universities, with the aim of providing a significant contribution to socio-economic development of the nation's innovation. . The population consisted of members and directors of research centers in the humanities core autonomous public universities in the state of Zulia. The data collection technique was the survey, with the application of a Likert questionnaire to a sample of 44 research professors of public universities autonomous state of Zulia. Previous results obtained as there is a moderate level of entrepreneurship to innovation in the study population, with a high level of attitude and attributes for entrepreneurship and a high range of technological skills to transform reality with innovative knowledge, but low level of funding and limited resources and a weak legal framework for the establishment of entrepreneurship to innovation.
\end{abstract}

Keywords: Entrepreneurial skills, Innovation, Public Universities, Development

\section{Desenvolvimento de competências empresariais e docentes de universidades públicas autónomas}

\begin{abstract}
RESUMO
Neste artigo, o desenvolvimento de competências empresariais e universidades públicas autônomas do estado Zulia, com base nas teorias de Auletta e Bridge (2010), Mas Torello (2011) e Hisrich, (2005) analisaram a pesquisa foi descritiva, não experimental e de campo. questionário do tipo Likert foi aplicado a uma amostra de 44 professorespesquisadores de universidades públicas autônomas do estado Zulia. Foi obtido como resultados anteriores de que existe um nível moderado de atitude e atributos para o empreendedorismo e uma alta gama de conhecimento tecnológico para transformar a realidade com conhecimento inovador, mas de baixo nível de financiamento e recursos escassos. Assim, propõe-se a crescer através de um modelo, o desenvolvimento de competências empresariais para a inovação em centros de pesquisa de ensino, a fim de transformar as universidades públicas autônomas nas instituições que produzem resultados tangíveis para o desenvolvimento sócio-produtiva a nação.
\end{abstract}

Palavras-chave: Competências empreendedoras, Innovación, Universidades Públicas Autónomas, Desenvolvimento socio-económico 


\section{Introducción}

En la actualidad, el nuevo orden mundial de la ciencia y la tecnología y los desafíos del siglo XXI, caracteriza un clima de turbulencia, escenarios económicos impredecibles que ameritan una cooperación orientada a fortalecer el mejoramiento de las capacidades intelectuales a fin de adaptarse a las exigencias del entorno.

En el ámbito de la Educación Superior, se coincide con el planteamiento de Puchmuller y Puebla (2014), al referirse a la posibilidad de acceder a la Sociedad de la Información (caracterizada por la creciente capacidad tecnológica para almacenar y difundir cada vez más) y el Conocimiento (caracterizada por la capacidad de apropiación crítica y selectiva de la información de los ciudadanos a fin de aprovechar esa información), con un cambio rotundo en la educación y en la participación de los individuos.

Ante lo planteado anteriormente, merece una revisión exhaustiva del papel de las universidades respecto a la formación de capacidades y transferencia de conocimientos que hasta ahora sigue prevaleciendo el formar profesionales para aspirar a un cargo o puesto de trabajo en organizaciones establecidas y se abandona la idea de crear negocios propios.

Asimismo, basándose en el aporte de la universidad en diversos países, se han analizado cuáles factores conllevan a que un país o grupo de países aliados impulsen o generen desarrollo sostenible y sustentable dentro del contexto geográfico, social, cultural y político. Según Leal (2012) la tecnología ha resultado ser una de las principales causas que ha permitido que los países que posean soberanía tecnológica alcancen niveles de progreso y desarrollo humano significativo. Venezuela, a pesar de contar con un marco regulatorio que fomenta la asimilación y desarrollo de nuevas tecnologías, no termina por generar crecimiento económico.

La poca información y difusión sobre políticas públicas orientadas a estimular el emprendimiento en el aparato productivo nacional, ha tenido como efecto que Venezuela se encuentre en los lugares más bajos de los mayores indicadores mundiales: el 122 de 139 en el Índice Global de Competitividad; el 102 de 121 del GII; y el 175 de 179 en el Índice de Libertad Económica, todo esto a pesar de ser un país bendecido por la naturaleza. Por lo que los indicadores mencionados anteriormente consideran múltiples variables entre las que se encuentran factores como marco jurídico, infraestructuras, capital humano y funcionamiento del mercado, entre otros. Según Auletta y Puente (2010) en Venezuela, los emprendedores representan apenas el 18 por ciento de la población adulta y la razón fundamental se debe a que "las nuevas empresas son iniciadas en industrias maduras y esencialmente con prácticas de negocios imitativas" (P. 28)

Actualmente, no se perfilan políti- cas para el desarrollo de competencias emprendedoras en el sector científico de las universidades públicas en la creación por ejemplo, de planes de negocios, transferencia tecnológica, creación de patentes, promoción de micro empresas innovadoras o productos de innovación social, ni otros planes conducentes a direccionar el desarrollo económico y social del país. Se deduce entonces, que los aportes y beneficios de las universidades a planes estratégicos del país son insuficientes y con bajo impacto.

Por lo que en el presente artículo, se plantean las siguientes interrogantes ¿Se puede desarrollar competencias emprendedoras en las universidades públicas autónomas?; y ¿Cuáles son las competencias del emprendimiento para la innovación en núcleos humanísticos de universidades públicas autónomas?

\section{Competencias del emprendi- miento para la innovación}

Ser competente, más que poseer un conocimiento formal, es la actividad desplegada en un contexto particular y el término es aplicado en la formación y perfeccionamiento de profesionales, entendiéndose como capacidad o conjunto de capacidades que incluyen el conocimiento y el uso del conocimiento.

Al respecto, Bedoya (2000) afirma que la competencia es apreciada como una potencialidad o una capacidad para resolver una situación problemática, explicar una solución y controlar y posicionarse en ésta. 
Por lo que la autora Almaguer (2014) propone diseñar estrategias de capacitación para el desarrollo de competencias de los trabajadores en diferentes sectores. La capacitación para Chiavenato (2009) es uno de los factores fundamentales para el desarrollo del talento humano, ya que capacita e incrementa el desarrollo profesional y personal de los empleados. En tanto, las competencias resumen las capacidades para integrar aquellos conocimientos adquiridos y rasgo de personalidad que permiten resolver situaciones diversas (Tejada, 1998).

La política de capacitación es una práctica cotidiana en toda organización que pretenda evolucionar en la actual sociedad de la información. Los últimos y novedosos avances tecnológicos obligan a las organizaciones, entre ellas las dedicadas a la formación y capacitación de talento humano, a desarrollar lineamientos, estrategias y procesos que sean favorables al desarrollo de las mismas.

Ahora bien, para lograr el desarrollo de competencias en busca de generar emprendimiento para la innovación, se debe entonces considerar el manejo de ventajas competitivas para impulsar la producción de contenidos científicos e innovaciones. Capacitar para innovar no debe reducirse exclusivamente a los aspectos técnicos e instrumentales, amerita una profundización de conocimiento para poder aplicarlo a la resolución de problemas complejos y
(2010) al explicar su posición de que la innovación está en las personas, plantean estimular el desarrollo de aquellas características que fomentan la creatividad y la generación de nuevas ideas.

Por otra parte, Drucker (2002), define a la innovación como la acción de dotar a los recursos con una nueva capacidad de producir riqueza así como también plantea que cualquier cambio en el potencial productor de riqueza ya existente, también es innovación. Drucker considera a la innovación como "herramienta específica de los empresarios; el medio por el cual explotar el cambio como una oportunidad para un negocio diferente".

Se infiere entonces que la innovación, lejos de parecerse a la inspiración, "es el resultado del trabajo duro, que no se centra en un «cierto tipo de personalidad», sino en el "compromiso en la práctica de la innovación»" (Drucker, 2002, p. 158)

En el ámbito educativo, el autor Mas Torelló (2011) hace mención sobre la "competencia innovadora", el cual se refiere al incremento del dominio competencial del profesor universitario en actividades de docencia, innovación e investigación. Complementa la tradicional formación teórica e investigadora en su propia área de conocimiento; capacitándole, en mayor medida, para la formación de nuevos profesionales y para contribuir a mejorar el corpus teórico y didáctico de su área de conocimiento, ya que los conocimientos y las competencias en innovación e investigación, tanto en su propia área (de la cual es experto) como del ámbito pedagógico, le permitirán realimentar su conocimiento (aplicándose tanto a la formación que pueda recibir como impartir), ayudándole ello a progresar por la senda de la calidad y por el camino hacia excelencia profesional.

Los estándares de competencias docente e investigativo de la UNESCO proponen aumentar la capacidad de innovar en la aplicación de Tecnologías de la Información y la Comunicación y establecen tres enfoques complementarios que son: nociones básicas de Tecnología de la Información y la Comunicación (TIC), profundización de conocimiento para poder aplicarlo a la resolución de problemas complejos y reales, y en tercer lugar la generación de conocimientos para, así como gestionar, producir y transferir conocimientos.

Sin embargo, se ha evidenciado en trabajos anteriores que la capacitación para el desarrollo de competencias en universidades públicas corresponde a la iniciativa propia de los investigadores y no del Centro de Investigación. La utilidad de esta capacitación se ha limitado a servicios de conectividad, selección de información y comunicación con otros. Por lo que se evidencia también una baja participación de la organización en gestionar la generación de competencias para beneficio de la propia organización (Viloria et. al.; 2012). 


\subsection{Competencias Empresariales}

Las competencias empresariales son el conjunto de habilidades, conocimientos, comportamientos, concepciones, valores, enmarcados dentro del paradigma educativo de la independencia, que busca formar una persona emprendedora, capaz de generar riqueza y desarrollo social, empezando por crear empresas hasta la formación de cadenas productivas, aprovechando las oportunidades, recursos y posibilidades que tenemos.

Si bien la competencia empresarial es requisito sine-quanon en el quehacer científico, se ha constituido en herramienta de éxito en naciones donde la investigación genera resultados tangibles e innovadores. La Toyota en Japón, o la Samsung en Korea del Sur, han registrado ganancias multimillonarias en los últimos 10 años gracias al desarrollo de competencias empresariales vinculadas con la Ciencia. A continuación se describen cuatro factores que pudieran desarrollar competencias empresariales las cuales son: Iniciativa empresarial, trabajo en equipo, visión de negocios y percepción de riesgos

\section{Iniciativa empresarial}

Cabe decir que innovación e iniciativa empresarial (IE) están estrechamente relacionadas, pero como afirman Auletta y Puente (2010) pocas iniciativas constituyen realmente innovaciones. "Las experiencias recientes de emprendedores venezolanos revelan que el grado de innovación de sus iniciativas depende, en gran medida, de sus habilidades y características personales" (P. 28).

IE se refiere entonces, a la idea, creación o propuesta del nacimiento de un negocio potencialmente exitoso, a partir de un sondeo -intencionado o no- del comportamiento del mercado interno y externo, lectura de indicadores de demanda social así como otras variables socioeconómicas que permitan a un sujeto o equipo de trabajo colocar un nuevo producto o servicio en el mercado. Se convierte en punto de partida para desarrollar una idea de negocio sustentado con estudio previo de las posibilidades de consolidarse dicha iniciativa.

La iniciativa empresarial, se perfila muy poco en la labor científica de universidades autónomas, ya que se evidencia una baja producción de contenidos significativos e innovadores, pues la producción intelectual de este sector se reduce a la tradición endogámica de crear y difundir contenidos casi exclusivamente a través de investigaciones formativas, con enfoques y métodos apegados al determinismo científico positivista; reducido nivel de convergencia, transdisciplinariedad y trabajo colaborativo. Es decir, son productos de investigación con muy poco potencial para el comercio.

\section{Capacidad de trabajo en equipo}

El trabajo en equipo suele definirse como: "un grupo de personas en busca de alcanzar un objetivo común" (Castillo y Quesada, 2001). Para lograr emprendimiento se hace necesario seleccionar personas comprometidas con el equipo (miembros del personal, en el caso de las organizaciones) y con interés de esforzarse para el logro de resultados colectivos.

Ahora bien, cuando las relaciones entre los miembros del personal son fluidas y se busca el consenso en situaciones de conflicto, se puede considerar que existe capacidad de trabajar en equipo. A juicio de De La Cruz (2014) la capacidad de trabajo en equipo es "la habilidad para colaborar e interactuar participando activamente en la consecución de un fin concreto" (P. 33). Además, expone De la Cruz que el trabajo en equipo necesita de compartir metas y anteponerlas a los propios intereses personales.

El autor Noe (2008) al igual que De la Cruz (2014) coinciden en plantear que para que se logre la capacidad de trabajar en equipo se debe desarrollar ciertas facultades en los individuos involucrados como: habilidades de comunicación necesarias para resolver la interrupción de las relaciones, mejorar la calidad de las decisiones y estimular la creatividad ante conflictos.

La capacitación del trabajo en equipo es esencial ya que "nuestra cultura se ha alimentado históricamente de logros individuales; con todo, las organizaciones usan equipos con mayor frecuencia" (Noe, 2008, p. 223)

En el ámbito de los centros de investigación, Solliero (2005) sostiene que 
la capacidad de trabajo en equipo se logra cuando: "un elevado nivel de integración organizacional puede mejorar la coordinación, la planificación y la puesta en práctica de estrategias de innovación" (P.86). Por otra parte, al autor afirma que esta integración organizacional puede originar resultados satisfactorios en sectores donde se generan conocimientos y tecnologías.

\section{Percepción de riesgos}

Asumir riesgos puede perfilarse como una capacidad emprendedora, sin embargo, es preciso aclarar lo descrito por Brunet (2009) al definir la toma de riesgos como acción en función de probabilidades de éxito. Estos riesgos para el emprendedor pudieran ser financieros, sociales o psicológicos (Hisrich, 2005).

Para Aponte (2003) la percepción de riesgos es una condición de instinto o cálculo de todo emprendedor ya que "el empresario tiende a asumir riesgos en la medida que es capaz de influir en el resultado de su acción". Por lo que según Ruesga (2012), la percepción del riesgo es una cuestión relativa: si una persona cree tener la habilidad suficiente para conseguir los objetivos propuestos, podrá también percibir los riesgos de fracaso de forma baja lo que le permitirá actuar ante tal percepción. Otro aspecto a resaltar es la capacidad de Visión de Negocio, el cual explica Auletta y Puente (2010), es cuando un emprendedor posee visión creativa que transforma en ideas de negocios situaciones y cambios del entorno visi-

\section{Actitud y atributos}

\section{Creatividad}

De acuerdo con Esquivias (2004) el proceso creativo es una de las potencialidades más elevadas y complejas de los seres humanos, éste implica habilidades del pensamiento que permiten integrar los procesos cognitivos menos complicados, hasta los conocidos como superiores para el logro de una idea o pensamiento nuevo. Considera Esquivias que la creatividad es un concepto que alude a uno de los procesos cognitivos más sofisticado del ser humano, todo apunta a que se encuentra influida por una amplia gama de experiencias evolutivas, sociales y educativas y su manifestación es diversa en un sin número de campos.

En la edición de (1992, p. 593) el Diccionario de la Real Academia de la Lengua Española define la creatividad como: "facultad de crear, capacidad de creación"

No obstante, la creatividad como concepto ha sido un tema no abordado y por lo mismo poco estudiado, es hasta años recientes donde surgen teóricos que se abocan a profundizar sobre el tema y se desarrollan trabajos y aportaciones alusivas a este concepto.

\section{Responsabilidad Social}

La Responsabilidad Social, a criterio del autor Urzùa (2001), se define como la orientación de las actividades individuales y colectivas en el sentido que permita a todos igual- dad de oportunidades para desarrollar sus capacidades, apoyando la eliminación de los obstáculos estructurales de carácter económico, social, culturales y políticos que afectan o impiden ese desarrollo.

Por lo que la Responsabilidad Social como proyecto se fundamenta en valores organizacionales y locales inmanentes pero sobre todo en valores universales trascendentes y por ello se ha convertido en una de las más importantes dimensiones éticas para toda organización.

En el seno de la universidad, Palencia y otros (2007) plantean que se debe definir la noción esencial de cultura y humanismo y construir, a partir de los más altos valores tales como el servicio, la colaboración el bienestar común, la equidad, la justicia, la responsabilidad, la honestidad y el manejo de la verdad, los principios e ideales que regirán su destino, amén del plan para ejecución.

\section{Liderazgo}

El liderazgo, segùn Kaplan y Norton (2004), es la capacidad de construir consenso frente a la incertidumbre. Propone Petit y Emilia (2007) la invención, como base del proceso innovador, requiere de estímulo gerencial mediante un liderazgo efectivo y de una gerencia emprendedora innovadora que promueva actos creativos en los procesos que se ejecutan en todas las estructuras productivas de los países.

Para Nieto Ariza (2015) el liderazgo es un factor influyente en cada ser 
humano, ya que posee talentos innatos o adquiridos que pueden propiciar actitudes de liderazgo en labores diarias que realicen con mayor frecuencia. "La clave para lograr liderazgos más asertivos está en la forma cómo lo transmiten y es allí donde la comunicación juega un papel fundamental" (Nieto Ariza, 2015, p.39)

El tema de liderazgo en organizaciones educativas ha sido un debate incansable en los últimos 50 años, debido a su impacto e importancia al momento de medir la calidad educativa, rendimiento académico así como el desarrollo y expansión de algunas universidades en el mundo. Los cargos directivos de toda institución universitaria, desde los jefes de departamento hasta las propias autoridades rectorales, no pueden seguirse considerando como plazas de ocupación burocrática con tareas monótonas sin metas ni proyectos alcanzables. Por el contrario, todo capital humano en puestos de dirección debe asumir el compromiso y la responsabilidad de generar resultados productivos y beneficiosos para la propia institución y su entorno.

Cuando la cabeza de una organización no presenta proyectos innovadores o emprendedores, no se comunica y atiende las inquietudes de sus subordinados, pero además, se limita a una operatividad de supervivencia básica y predecible, se está entonces ante una crisis de liderazgo.

Lo anterior ha sido un reflejo evidente en muchos centros de investigación en universidades autónomas del país, al menos en la consolidación de proyectos innovadores de trascendencia nacional e internacional.

\section{Competencias Tecnológicas}

En palabras de Cabello (2000, p. 1), las competencias tecnológicas surgen a partir de estrategias o diseños apoyados en el conocimiento "sobre el conjunto de los saberes previos con los que cuentan los destinatarios de las mismas y también sobre las actitudes con las cuales los sujetos se enfrentan con las tecnologías y afrontan los procesos de aprendizaje".

Echeverría, (2001), indica que las competencias virtuales representan el conjunto de conocimientos y de habilidades que necesitan los docentes e investigadores para desempeñar tareas relacionadas con las herramientas y estrategias tecnológicas, que permitan la participación activa en los procesos de investigación. Según Tulodzieki (1999), citado por Sevillano y Quicios (2009) las competencias virtuales son entendidas como la capacidad y las condiciones fundamentales en el tratamiento y procesos ligados a las tecnologías. En este espacio virtual se ofrecen realidades importantes para la planificación académica y aplicando software específicos.

Por otra parte, Viloria et. al. (2012), acuña el término "competencia digital" en la que exige una buena comprensión y amplios conocimientos sobre la naturaleza, la función y las oportunidades de las TIC en situaciones cotidianas de la vida profesional y social, además de requerir el uso de la información de manera crítica y sistemática, saber evaluar su pertinencia social y científica, diferenciar entre información real y virtual,

$\mathrm{Al}$ referirnos a las competencias Tecnológicas del profesorado universitario, esto implica en primer lugar plantearse qué se entiende por competencias tecnológicas, se considera como el conjunto de valores, creencias, conocimientos, capacidades actitudes y aptitudes que los investigadores de la Comunicación, tanto a título personal como colectivo, deben adquirir con el propósito de generar nuevos conocimientos y aplicaciones.

Hoy por hoy, el concepto de competencias se ha renovado gracias a la inclusión tecnológica en las diferentes áreas del conocimiento, no podemos mirar al investigador desde las competencias básicas y genéricas que en materia de formación obtuvo y en el ámbito de experiencia perfeccionó, adaptándola a los diversos cambios sociotecnológicos.

Asimismo, es esencial el desarrollo de habilidades tecnológicas puesto que la mayoría de los éxitos en acciones tecnológicas y espíritu emprendedor innovador relacionado con la tecnología, están desarrollados por un conjunto de personas, cuyos conocimientos tecnológicos experimentados juegan un papel fundamental en la organización para lograr un excelente manejo del espíritu emprendedor (Antoncic y Hisrich, 2001) 


\section{Apropiación social de las Tic}

Al referirse a la apropiación social de las tecnologías, Serge Proulx (2004, p. 26) lo define como "dominio técnico y cognitivo del artefacto". Proulx plantea además que la apropiación social de la tecnología se genera cuando se integra el objeto técnico en la práctica cotidiana del usuario y crea acciones encaminadas a generar novedad en la práctica social e impactar finalmente innovar.

Gomez Mont (2009) retoma la definición de apropiación de Proulx, quien considera que ésta tiene que ver con la integración significativa del objeto técnico en la actividad diaria del sujeto, la creación y finalmente, a un nivel colectivo de apropiación social generar innovación. En líneas generales, para que exista apropiación social, se debe hacer uso de bienes producidos por otras culturas y adaptarlas a nuestras realidades, dominarlas y obtener resultados beneficiosos.

Por otra parte, Gonzalo Morelis (2006) señala que la apropiación social de las TIC, en especial de Internet es la forma mediante la cual, esta herramienta adquiere un significado en la cotidianidad de los grupos sociales y se constituye en algo útil para la generación de nuevos conocimientos que permiten a los usuarios transformar la realidad en la cual se encuentran.

Serge Proulx (en Neuman, 2008) considera que se requieren cuatro condiciones para la apropiación so- cial de una tecnología: "a) El dominio técnico y cognitivo del artefacto; b) la integración significativa del objeto técnico en la práctica cotidiana del usuario; c) el uso repetido de esta tecnología que abre hacia posibilidades de creación (acciones que generan novedad en la práctica social); d) finalmente, a un nivel propiamente más colectivo, la apropiación social supone que los usuarios estén adecuadamente representados en el establecimiento de políticas públicas y al mismo tiempo sean tenidos en cuenta en los procesos de innovación (producción industrial y distribución comercial)".

Las últimas dos condiciones que estipula Proulx son las que diferencian la apropiación de otros conceptos como adaptación, uso, asimilación. Son dos condiciones a tener muy en cuenta en el discurso de las políticas públicas: la creación, la innovación en las prácticas sociales producto del uso, en este caso de las TIC y la implicación de esos usuarios en el establecimiento de políticas públicas.

No obstante, existe una línea de investigación crítica al tema de la apropiación social de las tics (Neüman, 2008, Morillo Maldonado, 2010, entre otros). Para la autora Neuman (2008), el término "apropiación social", nos precisa que en el ámbito de las tecnologías de la información y la comunicación, mucho se ha estudiado sobre la apropiación de las TICs en Venezuela, pero poco se ha abordado el significado de "hacer propio" en Latinoamérica lo desarrollado en países de primer mundo.
Además expone que las sociedades que habitan la periferia del sistema capitalista actual se mueven en espacios donde las estructuras y los productos sociales que las rodean son de origen exógeno: la "industria cultural", los objetos de consumo o mercancía y la estructura de las organizaciones formales.

Al respecto, Neuman asume el siguiente planteamiento crítico:

La "apropiación social" se asumiría como un proceso por medio del cual grupos sociales marginales del sistema económico capitalista interactúan con la propuesta cultural, económica, organizacional y de consumo de ese sistema mediante formas de adjudicación de nuevos sentidos, usos y propósitos que actúan como filtros y les permite mantener su propio horizonte de comprensión del mundo. (P. 71)

$\mathrm{Al}$ igual que Neuman, otros autores hacen referencia, desde una visión marxista, el concepto de apropiación social. Morillo Maldonado (2010) argumenta que la apropiación de las tics como causa de la modernidad en la que vivimos es producto de la globalización "alientante" en la sociedad que ha generado "la cosificación del ser humano". La modernidad (Mas Herrera, 2007, citado por Morillo M. 2010) "es un tiempo en el cual se cosifica la acción del ser humano dando predominio a los resultados materiales, tangibles, medibles $\mathrm{y}$ controlables" (P. 5).

Desde el punto de vista del impac- 
to cultural, sostienen Núñez y Ledezma (2007) que el uso de las Tics pasan por un proceso de adaptación social y cultural que los consumidores imprimen como rasgo distintivo por la interpretación que efectúan al momento de manejarlas.

\section{Metodología}

La investigación se ajusta a los parámetros tipificados como descriptivos, en cuanto a que "buscan especificar las propiedades, las características y los perfiles de personas, grupos, comunidades, procesos, objetos o cualquier otro fenómeno que se someta a un análisis" (Hernández et al 2006: 102). Además, el diseño de ésta investigación es de tipo no experimental, los datos serán recolectados una sola vez, en un momento determinado de acuerdo a la técnica a utilizar, por el investigador y posteriormente realizar la descripción y análisis.

Una vez identificada la población, la muestra y caracterizados los informantes, se procede a seleccionar las técnicas e instrumentos de recolección de datos, mediante los cuales se obtendrá la información necesaria para llevar a cabo la investigación, todo en correspondencia con el problema, los objetivos y el diseño de investigación. Para Hurtado (2010), las técnicas tienen que ver con los procedimientos o formas utilizadas para la recolección de los datos.

La presente investigación, considerada como cuantitativa, es definida por Arias (2006), como una técnica que pretende obtener información suministrada por una muestra de sujetos acerca de si mismos, o en relación con un tema en particular. Para la presente investigación, se aplicó la técnica de la encuesta, a través de un cuestionario con preguntas relacionadas al objeto de estudio, y autoadministrado individual. Este instrumento fue suministrado a todos los integrantes de la Muestra, quienes se identificaron como personal docente y de investigación con participación en proyectos de investigación y desarrollo.

La población como "conjunto finito o infinito de elementos o unidades de observación que se consideran en un estudio: nación, estados, grupos, comunidades, objetos, instituciones, asociaciones, actividades, acontecimientos, personas", según Rísquez (1999, p. 48), está conformada por 80 miembros entre directivos e investigadores del personal docente y de investigación en calidad de miembro de los centros de investigación en el núcleo humanístico de la universidad del Zulia, en condición de activo y con participación en proyectos de I+D como investigador principal o co-investigador responsable; así como los directivos de los diferentes centros siete (7) centros de investigación del núcleo humanístico de Luz. Se estima que la muestra objeto de estudio está conformada por 44 miembros de centros de investigación en el Núcleo Humanístico de universidades públicas autónomas del estado Zulia.

\section{Análisis y Resultados}

Se observa en la Tabla 1, que el promedio de indicador Iniciativa Empresarial el porcentaje mayor fue $34,09 \%$, que corresponde A Veces (AV), seguidamente Casi Siempre (CS) obtuvo 31,82\%, mientras que Casi Nunca (CN) registró 28,79\%, hasta el menor 5,30\% que corresponde a Nunca (N). Al analizar el indicador Capacidad de Trabajo en Equipo, se observó que el $46,97 \%$ de la población objeto de estudio, se ubicó en la alternativa de respuesta Casi Siempre (CS), seguido de un 40,91\% que respondieron Siempre (S), igualmente se obtuvo un $9,85 \%$ para A Veces (AV), mientras que otro 2,27\% se ubicó en Nunca (N). Para Percepción de Riesgos, en este indicador se obtuvo el más alto valor en la opción A Veces (AV) con 40,15\%, seguidamente la opción Casi Nunca (CN) con 28,79\% y con 27,27\% registró la opción Casi Siempre (CS). Finalmente, el indicador Visión de Negocio presentó el mayor porcentaje de respuesta en la opción A Veces (AV) con 51,52\%, seguido de Casi Nunca (CN) el cual respondieron 24,24\%, con 14,89\% la opción Casi Siempre (CS) y con 7,58 la opción Siempre (S).

En cuanto a la media de la subdimensión Competencias Empresariales se obtuvo de 3,29 de promedio, y se ubica en el baremo de categorías entre el Rango $(2,6$ - 3,4) que corresponde a una categoría de Nivel Intermedio. Los resultados anteriores permiten afirmar que existe una alta capacidad de trabajo en equipo, 
lo cual coinciden tanto con Kaplan \& Norton (2004) como con Drucker (2002) en la premisa de que el éxito de una organización depende de la participación completa de todos sus miembros ya que a la larga genera: más utilidades, crecimiento sostenido y competencia eficaz.

También, puede compararse con los resultados de Palmeth (2015) al afirmar en su estudio que existen excelentes relaciones interpersonales con el personal que le rodea, casi siempre se promueve la cooperación y se tiene en cuenta la opinión de los demás miembros de la organización.

En la tabla Nro 2 de la subdimen- sión Actitud y Atributos, existe una tendencia mediana hacia una alta frecuencia en la mayoría de los indicadores. Se observa, por ejemplo, en el indicador Creatividad, la opción 3 (A Veces) con 40,91\% de respuesta el cual fue el porcentaje mayor. La opción 2 (casi siempre con 39,39\%) fue la segunda opción de mayor porcentaje, mientras que la opción Siempre (S) obtuvo 15,15\% en dicho indicador. Por otra parte, la Proactividad obtuvo $34,89 \%$ tanto en la opción Casi Siempre (CS) como en la opción A Veces $(\mathrm{AV})$ y en la opción Siempre se registró un $12,12 \%$, en tanto que la opción Casi Nunca $(\mathrm{CN})$ registró 17,42\% como la cuarta opción de mayor valor.
Para el indicador Responsabilidad Social, se obtuvo el más alto valor en la opción Siempre (S) con 29,55\%, seguido de Casi Nunca (CN) con 28,79\%, la opción Casi Siempre (CS) registró 21,21\% y A Veces (AV) con $19,70 \%$, seguidamente la opción Nunca con apenas 0,76\%. En cuanto a Liderazgo se generó un resultado que favorece a la alta frecuencia, ya que los niveles Intermedio, Alto y Muy Alto totalizaron un $71 \%$ de las opciones de respuesta distribuidos en 33,33\% para Casi Siempre (CS), 21,97 A Veces (AV) y 15,91 en la opción Siempre (S), sin embargo, la opción Casi Nunca (CN) obtuvo 20,45\% y la opción Nunca (N) un 8,33\%.

TABLA 1

\begin{tabular}{|c|c|c|c|c|c|c|c|c|c|c|c|c|c|}
\hline \multicolumn{14}{|c|}{ Dimensión: • Competencias para el emprendimiento } \\
\hline \multicolumn{14}{|c|}{ Sub Dimensión: Competencias Empresariales } \\
\hline Alternativa & \multicolumn{2}{|c|}{ TA } & \multicolumn{2}{|c|}{ MA } & \multicolumn{2}{|c|}{ NN } & \multicolumn{2}{|c|}{$\mathrm{MD}$} & \multicolumn{3}{|c|}{$\mathrm{TD}$} & & $\begin{array}{l}\text { Media } \\
\text { Ind. }\end{array}$ \\
\hline Indicador/valor & \multicolumn{2}{|c|}{5} & \multicolumn{2}{|c|}{4} & \multicolumn{2}{|c|}{3} & \multicolumn{2}{|c|}{2} & \multicolumn{2}{|c|}{1} & $\mathrm{Fa}$ & $\mathrm{Fr} \%$ & \\
\hline Iniciativa empresarial & 0,00 & 0,00 & 14,00 & 31,82 & 15,00 & 34,09 & 12,67 & 28,79 & 2,33 & 5,30 & 44,00 & 100,00 & 2,92 \\
\hline $\begin{array}{c}\text { Capacidad de trabajo } \\
\text { en equipo }\end{array}$ & 18,00 & 40,91 & 20,67 & 46,97 & 4,33 & 9,85 & 0,00 & 0,00 & 1,00 & 2,27 & 44,00 & 100,00 & 4,24 \\
\hline Percepción de riesgos & 0,67 & 1,52 & 12,00 & 27,27 & 17,67 & 40,15 & 12,67 & 28,79 & 1,00 & 2,27 & 44,00 & 100,00 & 2,97 \\
\hline Visión de negocio & 3,33 & 7,58 & 6,33 & 14,39 & 22,67 & 51,52 & 10,67 & 24,24 & 1,00 & 2,27 & 3,33 & 7,58 & 3,01 \\
\hline & & & & & & & & & & Medi & Subd & nensión & 3,29 \\
\hline
\end{tabular}


TABLA 2

\begin{tabular}{|c|c|c|c|c|c|c|c|c|c|c|c|c|c|}
\hline \multicolumn{14}{|c|}{ Dimensión: • Competencias para el emprendimiento } \\
\hline \multicolumn{14}{|c|}{ Sub Dimensión: Actitud y Atributos } \\
\hline Alternativa & \multicolumn{2}{|c|}{$\begin{array}{c}\mathrm{S} \\
\mathrm{TA}\end{array}$} & \multicolumn{2}{|c|}{$\begin{array}{l}\text { CS } \\
\text { MA }\end{array}$} & \multicolumn{2}{|c|}{$\begin{array}{l}\mathrm{AV} \\
\mathrm{NN}\end{array}$} & \multicolumn{2}{|c|}{$\begin{array}{l}\mathrm{CN} \\
\mathrm{MD}\end{array}$} & \multicolumn{2}{|c|}{$\begin{array}{l}\mathrm{N} \\
\mathrm{TD}\end{array}$} & & & $\begin{array}{l}\text { Media } \\
\text { Ind. }\end{array}$ \\
\hline Indicador/valor & \multicolumn{2}{|c|}{5} & \multicolumn{2}{|c|}{4} & \multicolumn{2}{|c|}{3} & \multicolumn{2}{|c|}{2} & \multicolumn{2}{|c|}{1} & Fa & $\mathbf{F r} \%$ & \\
\hline Creatividad & 6,67 & 15,15 & 17,33 & 39,39 & 18,00 & 40,91 & 1,00 & 2,27 & 1,00 & 2,27 & 44,00 & 100,00 & 3,63 \\
\hline Pro-actividad & 5,33 & 12,12 & 15,00 & 34,09 & 15,00 & 34,09 & 7,67 & 17,42 & 1,00 & 2,27 & 44,00 & 100,00 & 3,36 \\
\hline $\begin{array}{l}\text { Responsabilidad } \\
\text { Social }\end{array}$ & 13,00 & 29,55 & 9,33 & 21,21 & 8,67 & 19,70 & 12,67 & 28,79 & 0,33 & 0,76 & 44,00 & 100,00 & 3,42 \\
\hline Liderazgo & 7,00 & 15,91 & 14,67 & 33,33 & 9,67 & 21,97 & 9,00 & 20,45 & 3,67 & 8,33 & 44,00 & 100,00 & 3,13 \\
\hline $\begin{array}{c}\text { Compromiso con } \\
\text { el cambio }\end{array}$ & 17,00 & 38,64 & 20,33 & 46,21 & 4,67 & 10,61 & 1,00 & 2,27 & 1,00 & 2,27 & 44,00 & 100,00 & 4,17 \\
\hline Perseverancia & 18,67 & 42,42 & 24,33 & 55,30 & 0,00 & 0,00 & 0,00 & 0,00 & 1,00 & 2,27 & 44,00 & 100,00 & 4,36 \\
\hline & & & & & & & & & & & $\underset{\text { bbdir }}{\mathrm{Mc}}$ & sión & 3,68 \\
\hline
\end{tabular}

Para el indicador Compromiso con el Cambio, presentó el mayor porcentaje de respuesta la opción Casi Siempre (CS) $46,21 \%$, seguido la opción Siempre (S) con 38,64\%, luego se registró un $10,61 \%$ en la opción A Veces (AV) y finalmente la opción Casi Nunca (CN) y Nunca (N) obtuvieron similar resultado con 2,27\% cada uno. Finalmente, en el indicador Perseverancia, se tiene que las opciones Casi Siempre (CS) y Siempre (S) totalizaron un $98 \%$ de porcentaje de respuesta, siendo el resultado de más alta frecuencia en la presente subdimensión, de los cuales 55,3\% respondió Casi Siempre y el 42,42\% la opción Siempre (S). Ahora bien, al hacer referencia a la media de la subdimensión Actitud y Atributos, se registró un 3,68, lo cual se ubica en el baremo de categorías entre el Rango $(3,4-4,2)$ que corresponde a la categoría Alto.

Para la tabla Nro. 3, se observó en la subdimensión Competencias Tecnológicas, una alta tasa de respuesta hacia las categorías Siempre (S) y casi siempre (CS). Se tiene que en el indicador Apropiación Social de las Tic, se registró 59,85\% para la opción Casi Siempre (CS) y 32,58\% en la opción Siempre (S), totalizando un
93\% en estas dos categorías; le sigue la opción A Veces (AV) con 3,03\% y Casi Nunca $(\mathrm{CN})$ y Nunca $(\mathrm{N})$ con 2,27\% cada uno. En el indicador $\mathrm{Ca}-$ pacidad Tecnológica obtuvo un total de 34,09\% en la opción Casi Siempre (CS), le sigue la opción Siempre (S) con 29,55\%, la opción Casi Nunca registró el tercer mayor porcentaje con $21,21 \%$ y A Veces (AV) obtuvo $12,88 \%$. No obstante, en el indicador Asimilación Tecnológica se obtuvo como resultados que la opción Casi Siempre (CS) registró 43,28\% siendo el mayor porcentaje de este indicador, seguido de $28,79 \%$ de la opción A Veces (AV), la opción Casi Nunca 
TABLA 2

\begin{tabular}{|c|c|c|c|c|c|c|c|c|c|c|c|c|c|}
\hline \multicolumn{14}{|c|}{ Dimensión: Competencias para el emprendimiento } \\
\hline \multicolumn{14}{|c|}{ Sub Dimensión: Competencias Tecnológicas } \\
\hline Alterntativa & \multicolumn{2}{|c|}{$S$} & \multicolumn{2}{|c|}{ CS } & \multicolumn{2}{|c|}{$\mathrm{AV}$} & \multicolumn{2}{|c|}{$\mathrm{CN}$} & \multicolumn{2}{|c|}{$\mathrm{N}$} & & & \\
\hline & \multicolumn{2}{|c|}{ TA } & \multicolumn{2}{|c|}{ MA } & \multicolumn{2}{|c|}{ NN } & \multicolumn{2}{|c|}{ MD } & \multicolumn{2}{|c|}{$\mathrm{TD}$} & & & $\begin{array}{l}\text { Media } \\
\text { Ind. }\end{array}$ \\
\hline Indicador/valor & \multicolumn{2}{|c|}{5} & \multicolumn{2}{|c|}{4} & \multicolumn{2}{|c|}{3} & \multicolumn{2}{|c|}{2} & \multicolumn{2}{|c|}{1} & Fa & $\mathrm{Fr} \%$ & \\
\hline $\begin{array}{l}\text { Apropiación social } \\
\text { Tic }\end{array}$ & 14,33 & 32,58 & 26,33 & 59,85 & 1,33 & 3,03 & 1,00 & 2,27 & 1,00 & 2,27 & 44,00 & 100,00 & 4,18 \\
\hline $\begin{array}{l}\text { Capacidad } \\
\text { Tecnológica }\end{array}$ & 13,00 & 29,55 & 15,00 & 34,09 & 5,67 & 12,88 & 9,33 & 21,21 & 1,00 & 2,27 & 44,00 & 100,00 & 3,67 \\
\hline $\begin{array}{l}\text { Asimilación } \\
\text { Tecnológica }\end{array}$ & 4,67 & 10,61 & 19,00 & 43,18 & 12,67 & 28,79 & 6,67 & 15,15 & 1,00 & 2,27 & 44,00 & 100,00 & 3,45 \\
\hline & & & & & & & & & & Medi & Subdi & ensión & 3,77 \\
\hline
\end{tabular}

(CN) obtuvo 15,15\% y finalmente la opción Siempre (S) totalizó 10,61\%. Para la tabla 3 en resumen, la subdimensión Competencias Tecnológicas registró una media de 3,77 de respuesta, ubicándose entre el $(3,4-4,2)$ que corresponde a la categoría Alto.

\section{Conclusiones}

A partir de los resultados anteriores, se puede determinar que los centros de investigación del núcleo humanístico de universidades públicas autónomas producen conocimientos para fines de divulgación científica, transferencia de conocimiento y cooperación nacional e internacional., pero con moderados niveles de iniciativa emprendedora para fines in- delo de gestión de emprendimiento para la innovación.

Pese a registrarse un bajo nivel de financiamiento así como recursos escasos para actividades innovadoras, existen .unos moderados niveles de actitud y atributos para el emprendimiento y un alto rango de competencias tecnológicas para generar conocimientos innovadores, lo que permitiría transformar las universidades públicas autónomas en instituciones que realmente promuevan, desarrollen y lideren la innovación.

Es decir, el tema del emprendimiento para la innovación se desliga de las tareas cotidianas existentes en nuestras universidades públicas, lo que desvirtúa cualquier iniciativa emprendedora sustentada, por ejemplo, en la creación de nuevos modelos de negocios que pudieran generar empleo, crecimiento económico y calidad de vida.

Se destaca por, ejemplo, alta disposición para la creatividad, proactividad y compromiso con el cambio, elementos importantes para el desarrollo del emprendimiento para la innovación. Pero otros indicadores como iniciativa empresarial, percepción de riesgos y visión de negocio registraron unos resultados moderados o bajos, lo que significa que son elementos para el emprendimiento poco desarrollados en el personal docente y de investigación encuestado. 
Por lo que se hace urgente construir un modelo de emprendimiento para la innovación en universidades públicas autónomas para generar una cultura innovadora con la generación de ideas emprendedoras. Dicho modelo de emprendimiento para la innovación, intenta desarrollar las competencias emprendedoras para compromiso con el cambio en los miembros de centros de investigación en núcleos humanísticos de universidades públicas autónomas, fomentando una actitud con iniciativa empresarial, creatividad, responsabilidad social, visión de negocios, y percepción de riesgos que permita el desarrollo de productos de innovación empresarial o social, explotando el cambio como una oportunidad para un negocio diferente.

El modelo de emprendimiento para la innovación en universidades públicas autónomas deberá atender aspectos como:

Convertir a las universidades públicas autónomas bajo una cultura emprendedora, que respondan de manera coherente, estratégica y oportuna ante las exigencias que este les hace, sin que ello signifique una amenaza para su misión académica tradicional.

Estimular la acción humana en el personal docente y de investigación de las universidades estudiadas, con el fin de desarrollar oportunidades rentables bajo riesgo basado en las demandas y necesidades para tener la posibilidad de crear o mejorar un producto, un proceso o un sistema a partir de tecnologías existentes.

Tomar en cuenta la capacidad en el uso de las tics por el personal docente y de investigación del núcleo humanístico para desarrollar proyectos emprendedores e innovadores, ya que a pesar de poseer altas habilidades en el manejo de las tecnologías, no se manifiesta aún en productos de innovación.

Con el modelo de emprendimiento para la innovación, se intenta desarrollar compromiso con el cambio en los miembros de centros de investigación en núcleos humanísticos de universidades públicas autónomas, para así brindar más aportes a la ya precaria situación económica en el país.

\section{Referencias}

Almaguer M., Maida L. (2014.)Estrategia de capacitación para desarrollar competencias laborales. Recuperado el 20/08/2014 de http:/ / www.gestiopolis.com/estrategiade-capacitacion-para-desarrollarcompetencias-laborales/

Auletta, N. y Puente, R. (2010) ¿Qué hace a un emprendedor innovador? DEBATES IESA • Volumen XV • Número 2. http://www.itesm.mx/ Consultado el 15/07/2015

Bedoya, J. (2000). Epistemología y pedagogía. Editorial Graó. Barcelona, España.

Brunet, L. (2009). El Clima de Trabajo en las Organizaciones. Definición, diagnóstico y consecuencias. Edito- rial Trillas. México.

Cabello, R. (2000) Aproximación al Estudio de Competencias Tecnológicas. Universidad Nacional de Córdoba. Córdoba, Argentina.

Castillo, E. y Quesada, C. (2001) Manual de Capacitación para Emprendimientos Rurales. IICA Agencia ASDI. San José, Costa Rica

DE la Cruz Lablanca, Ignacio (2014) Comunicación efectiva y trabajo en equipo. Colección Aula Mentor. Madrid, España

Drucker, P. (2002): The discipline of innovation. The innovative enterprise. Harvard Business Review. Consultado el 10/05/2015

Esquivias S., María Teresa (2004) Revista Digital Universitaria. Creatividad: definiciones, antecedentes y aportaciones. Volumen 5 Número 1. ISSN: 1067-6079

Gómez Mont, C. (2009) Sociedad del conocimiento y apropiación tecnológica: Algunos postulados para pensar la diversidad cultural desde la cosmovisión indígena en México. Memorias del Coloquio Científico Internacional De la Sociedad de la Información a las Sociedades del Conocimiento. Redes y Comunidades de Saberes Compartidos. 1-15. Recuperado de http://132.248.192.201/ memoires/pdf2/e_10.pdf

Gonzalo, Morelis (2006): Déficit de acceso y apropiación social de las tecnologías de la información y comunicación. Fundación Escuela de 
Gerencia Social. Caracas-Venezuela.

Hernández, S. (2010). Metodología de la Investigación. México: Impreso en Grupo Infagon PROULX, Serge (2004). La Révolution Internet en question. Québec, Amérique. Québec, Canadá.

Hisrich, R.D. (1988-1990): Entrepreneurship Development in public Enterprises. Joseph Prokopenko, Igor Pavlin, International Labour Organization, 1991.

Kaplan, R. S. \& Norton, D. P. (2004). Strategy Maps: converting intangible assets into tangible outcomes. Harvard: Harvard Business School Publishing.

MAS Torelló, Òscar (2011) El Profesor Universitario: Sus Competencias y Formación. En revista. Profesora. VOL. 15, 3. Barcelona, España

Morillo Maldonado (2010) Apropiación social de las Tecnologías de la Información y Comunicación por activadores culturales de Maracaibo en el periodo 2006- 2010. III Congreso InveCom 2011. En: www.congresoinvecom.org

Neuman, M. (2008). Construcción de la categoría "Apropiación social". Revista QUÓRUM ACAÉMICO, Vol. 5, N 2. Universidad del Zulia, Maracaibo, Venezuela. Disponible en: http:// revistas.luz. edu.ve/index.php / quac/article / viewFile/ 2650/2564 [Consultado el 20/05/2013].
Nieto Ariza, M. (2015). La Comunicación, herramienta de construcción de liderazgos en organizaciones sociales de mujeres afrodescendientes del Caribe colombiano. Revista Encuentros, Universidad Autónoma del Caribe, 13 (1), pp. 37-46. DOI: http:/ / dx.doi.org/10.15665/ re.v13i1.347

Palencia, Jorge \& cols. (2007) La responsabilidad social: un proyecto prioritario en la agenda universitaria para lograr su transformación. VI Jornadas Nacionales de Investigación Humanística y Educativa. Postgrado FHE-LUZ. Octubre 2007. Maracaibo, Venezuela

Palmeth, Mónica (2015) “Modelo de Cultura de Emprendimiento en Instituciones con Ciclos Propedeuticos en el Departamento del Atlántico (Colombia)". Universidad Dr. Rafael Belloso Chacín Doctorado en Ciencias: Mención Gerencia. Maracaibo, Venezuela

Petit Torres, Elsa Emilia (2007) La gerencia emprendedora innovadora como catalizador del emprendimiento económico. Revista de Ciencias Sociales (RCS) Vol. XIII, No. 3, Septiembre - Diciembre 2007, pp. 495 506

Puchmüller, A.; Puebla, M. (2014). TIC en Educación Superior: usos e implicancias en dos carreras de instituciones argentinas. Revista Encuentros, Universidad Autónoma del Caribe, 12 (2), pp. 11-23

Ruesga Pons, Juan Pablo (2012) Em- prendimiento empresarial, miedo y resiliencia en el sector papelero. Universidad Dr. Rafael Belloso Chacín Doctorado en Ciencias Gerenciales. Maracaibo, Venezuela

Solleiro, J. et al. (2009). Gestión del conocimiento en Centros de Investigación y Desarrollo de México, Brasil y Chile; Programa de Investigación sobre Economía del Conocimiento en América Latina y el Caribe, Facultad Latinoamericana de Ciencias Sociales, FLACSO- IDRC, México

Tejada, J. (1998). El desarrollo y la gestión de competencias profesionales: una mirada desde la formación. Revista Iberoamericana de educación. Recuperado el 14 de agosto de 2011 de: http://www.rieoei.orh/ deloslectores / 1089tejada

Urzúa, Raúl. (2001) La Responsabilidad Social de las Universidades: Una Guía de Discusión. Documento presentado en el Taller "Elaboración de Estrategia común para la Expansión de la Responsabilidad Social en las Universidades Chilenas". Disponible en http: / / www.construyepais. $\mathrm{cl} /$ documentos / larsdelasuniversidadesrurzua.PDF

Viloria Hender, Villalobos F., Espinosa A. (2012) Redes sociales, competencias tecnológicas y producción intelectual de los posgrados en Comunicación venezolanos. En revista Encuentros, Vol. 11, No. 1. Universidad Autónoma del Caribe, Colombia. 